\title{
Nonlinear bending behavior of EMC laminates at elevated temperatures
}

\author{
Zhiyuan Xiong ${ }^{\text {a }}$, Rui Wu, Ruixiang Song, Zhongxu Kang, Huijuan Zhang and Qiang Liu \\ Beijing Municipal Institute of Labour Protection, Beijing 100054, China
}

\begin{abstract}
Elastic memory composite (EMC) is shape memory polymer reinforced by continuous fibers. When environmental temperature of EMC is above the glass transition temperature of its resin $(\mathrm{Tg})$ the resin is in the rubbery state and with high compliance, which makes EMC realize high packaging strain through microbuckling of the compressed fibers. In this study, the neutral axis location and fiber microbuckling half wavelength of EMC laminate are firstly introduced and shortages that exist in present literature are also perfected. Secondly, the known neutral axis location and half wavelength expressions are substituted into the total strain energy expression of packaged EMC laminate and then according to the principal of virtual work the total strain energy expression is deduced to yield the nonlinear relationship between bending moment and curvature of EMC laminate. Furthermore, the theoretical curve of nonlinear bending behavior is basically agreeable with the 4-point pure bending testing result of EMC laminate in their applicable bending range at elevated temperature.
\end{abstract}

Keywords: elastic memory composite, neutral axis, half wavelength, microbuckling.

\section{Introduction}

Elastic memory composite (EMC) is composed of shape memory polymer [1,2] matrix and continuous fibers reinforcements. Experimental study has confirmed that the matrix is in the rubbery state and with high compliance above the glass transition temperature of its resin $(T g)$, which makes EMC realize high packaging strain through microbuckling of the compressed fibres [3-6]. When the environmental temperature decreases lower than $\mathrm{Tg}$ EMC can keep the packaging shape by itself and a reheating of the EMC above $\mathrm{Tg}$ will make it recover to the original geometry [7-9]. EMC has not only high structural properties such like traditional continuous fiber reinforced polymer-base composites, but also very high bending capability and shape memory characteristics. Hence, this type of functional materials has great potentials in future space deployable structures [10-14].

The work of Francis et al [15] is the starting point of this investigation. On the basis of their study, the following work will be continued in this paper.

Firstly, when a unidirectional EMC laminate is bent above $T g$ its neutral axis location and the fiber microbuckling half wavelength are introduced, and the shortages that exist in present literature [15] are pointed out and perfected. Secondly, the known neutral axis location and half wavelength expressions are substituted into the total strain energy expression of bending EMC laminate. According to the principal of virtual work, the total strain energy is deduced to yield the nonlinear 
relationship of bending moment-curvature for EMC laminate. The theoretical curve is basically agreeable with the 4-point pure bending testing result of EMC laminate above $T g$.

\section{Neutral axis location}

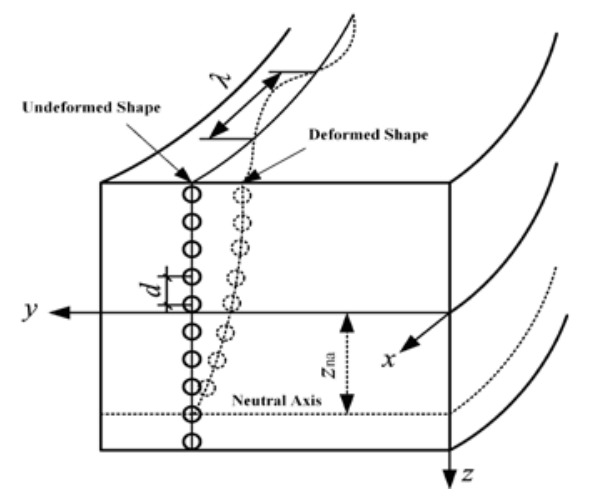

Figure 1. A sketch of a unidirectional EMC laminate under bending.

Fig. 1 is a sketch of a unidirectional EMC laminate under bending above $T g$. The length, width and thickness of EMC laminate are respectively 1, w, t. When an EMC laminate is bent above $T g$ the effective axial compressive stiffness in compressive zone is much less than the axial tensile stiffness in tensile zone due to the fiber microbuckling and matrix softness. To keep equilibrium of the internal forces for EMC laminate the neutral axis must shift toward the tensile zone. Based on the neutral axis EMC laminate is divided compressive and tensile zones and it is assumed that all of the fibers in the compressive zone occur microbuckling. The xyz coordinate system is shown in Fig.1 and the neutral axis location zna is the distance from the y axis or geometric midplane to neutral axis. All the half wavelengths $\lambda$ of sinusoidal-like microbuckling fibers in compressive zone are basically equivalent.

The neutral axis location zna is expressed as [15]

$$
z_{n a}=\frac{t}{2}+\frac{1}{\kappa} \frac{G_{x y}}{E_{x}}-\frac{1}{\kappa} \sqrt{\left(\frac{G_{x y}}{E_{x}}\right)^{2}+2 t \kappa\left(\frac{G_{x y}}{E_{x}}\right)}
$$

where $k, E_{\mathrm{x}}$ and $G_{\mathrm{xy}}$ respectively denote the bending curvature, longitudinal Young's modulus and in-plane shear modulus of EMC laminate. Utilizing the relationship between nominal bending $\operatorname{strain}_{\varepsilon_{\text {nom }}}$ and curvature $\kappa$ of the laminate, $\varepsilon_{\text {nom }}=t \kappa / 2$, after eliminating $\kappa$ Equation (1) can be expressed as

$$
\frac{z_{n a}}{t}=\frac{1}{2}\left[1+\frac{1}{\varepsilon_{\text {nom }} \frac{E_{x}}{G_{x y}}}\left(1-\sqrt{1+4 \varepsilon_{\text {nom }} \frac{E_{x}}{G_{x y}}}\right)\right]
$$


Table 1. Structural parameters and mechanical properties of EMC specimen $(\mathrm{T}>\mathrm{Tg})$ [15].

\begin{tabular}{lccl}
\hline Parameter & Value & Unit & \multicolumn{1}{c}{ Description } \\
\hline$t$ & 1.0 & $\mathrm{~mm}$ & Thickness of laminate \\
$w$ & 25 & $\mathrm{~mm}$ & Width of laminate \\
$l$ & 100 & $\mathrm{~mm}$ & Length of laminate \\
$h$ & $5.2 \times 10^{-6}$ & $\mathrm{~m}$ & Fiber diameter \\
$d$ & $7.3 \times 10^{-6}$ & $\mathrm{~m}$ & Distance of two adjacent fiber centrals \\
$I_{f}$ & $3.59 \times 10^{-23}$ & $\mathrm{~m}^{4}$ & Fiber area moment of inertia \\
$V_{\mathrm{f}}$ & 0.4 & - & Fiber volume fraction \\
$E_{\mathrm{x}}$ & 110 & $\mathrm{GPa}$ & longitudinal Young's modulus of \\
$G_{\mathrm{xy}}$ & 67.4 & $\mathrm{MPa}$ & laminate \\
$G_{\mathrm{yz}}$ & 8.87 & $\mathrm{MPa}$ & shear modulus in x-y plane of laminate \\
$E_{\mathrm{f}}$ & 276 & $\mathrm{GPa}$ & Fiber Young's modulus \\
\hline
\end{tabular}

Table 1 is the structural parameters and mechanical properties of EMC laminate specimen above $T_{\mathrm{g}}[15]$. Substituting the related values in Table 1 into Equation (2) and Fig.2 can be determined as the following.

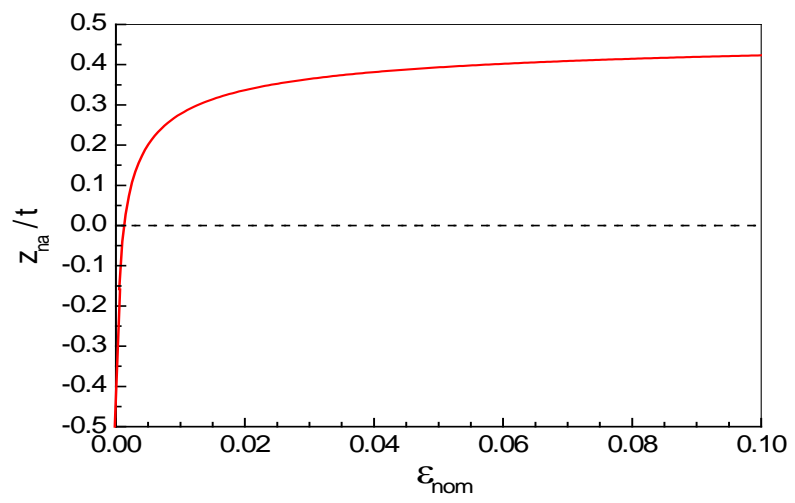

Figure 2. Shifting of neutral axis with the increasing of bending level.

\section{Half wavelength}

Francis et al deduce the individual strain energy components and the total energy stored in the EMC laminate [15].

$$
\begin{gathered}
U_{x x}=\frac{1}{48} E_{x} \operatorname{lw}\left(t-2 z_{n a}\right)^{3} \kappa^{2} \\
U_{x y}=\frac{1}{2} G_{x y} l w \kappa\left(\frac{t}{2}+z_{n a}\right)^{2} \\
U_{y z}=\frac{G_{y z} w l \kappa \lambda^{2}}{4 \pi^{2}} \ln \frac{2\left(t+2 z_{n a}\right)}{d} \\
U_{f}=\frac{\pi l w V_{f} E_{f} I_{f}}{2 h^{2} \lambda^{2}} \kappa\left(t+2 z_{n a}\right)^{2} \\
U_{t}=\frac{1}{48} E_{x} l w\left(t-2 z_{n a}\right)^{3} \kappa^{2}+\frac{1}{2} G_{x y} l w \kappa\left(\frac{t}{2}+z_{n a}\right)^{2} \\
+\frac{G_{y z} w l \kappa \lambda^{2}}{4 \pi^{2}} \ln \frac{2\left(t+2 z_{n a}\right)}{d}+\frac{\pi l w V_{f} E_{f} I_{f}}{2 h^{2} \lambda^{2}} \kappa\left(t+2 z_{n a}\right)^{2}
\end{gathered}
$$


Where $E_{x}, G_{x y}, G_{y z}$ respectively denote longitudinal Young's modulus, shear modulus in $x-y$ and $y$ $z$ planes of EMC laminate; $E_{\mathrm{f}} I_{\mathrm{f}}, V_{\mathrm{f}}, h$ respectively are the flexural stiffness, volume fraction, diameter of the fiber; $d$ is the distance of two adjacent fiber centrals; $U_{x x}$ is the extension strain energy in the tensile zone, $\mathrm{U}_{\mathrm{xy}}$ is the shear strain energy produced in $x-y$ plane of the compressive zone, $\mathrm{U}_{\mathrm{yz}}$ is the shear strain energy produced in $y-z$ plane of the compressive zone, $\mathrm{U}_{\mathrm{f}}$ is the bending strain energy of microbuckling fibers, $U_{t}$ is the total strain energy that is composed of the above four strain energy components.

The neutral axis location $z_{\text {na }}$ and half wavelength $\lambda$ are two unknown variables in these equations. The expression of neutral axis location $z_{\mathrm{na}}$ has determined in Equation (1). Equation (1) is substituted into the total strain energy, Equation (7), and the minimized $U_{t}$ can be obtained by differentiating the below equation with respect to $\lambda$.

$$
\begin{aligned}
& \frac{\partial U_{t}}{\partial \lambda}=\frac{\partial\left(U_{y z}+U_{f}\right)}{\partial \lambda}=0 \\
& \lambda=\pi \sqrt[4]{\frac{V_{f} E_{f} h^{2}\left(t+\frac{1}{\kappa} \frac{G_{x y}}{E_{x}}-\frac{1}{\kappa} \sqrt{\left.\left(\frac{G_{x y}}{E_{x}}\right)^{2}+2 t \kappa \frac{G_{x y}}{E_{x}}\right)^{2}}\right.}{8 G_{y z} \ln \frac{4 t+\frac{4}{\kappa} \frac{G_{x y}}{E_{x}}-\frac{4}{\kappa} \sqrt{\left(\frac{G_{x y}}{E_{x}}\right)^{2}+2 t \kappa \frac{G_{x y}}{E_{x}}}}{d}}}
\end{aligned}
$$

But $z_{\text {na }}$ is hypothesized to be $t / 2$ for deducing the half wavelength $\lambda$ in the reference ${ }^{[11]}$, which means that the neutral axis locates the tensile surface of packaged EMC laminate from beginning to end and the distance between the neutral axis and the tensile surface is ignored. The calculated result is ${ }^{[15]}$

$$
\lambda_{c r}=\left(\frac{9 \pi^{3} V_{f} t^{2} E_{f} I_{f}}{2 h^{2} \ln \left(\frac{3 t}{d}\right) G_{y z}}\right)^{\frac{1}{4}}
$$

It is too simplified that $z_{n a}$ is directly equal to $t / 2$, because Fig. 2 shows that the distance of the neutral axis and tensile surface is slightly less than ten of percent of the thickness of EMC laminate $t$ when nominal strain $\varepsilon_{\text {nom }}$ is greater than 0.06 and the distance is fairly large and varies from $0.5 t$ to $0.1 t$ when nominal strain $\varepsilon_{\text {nom }}$ is less than 0.06 .

Substituting the related values in Table 1 into Equations (9) and (10), Fig.3 can be plotted as the following.

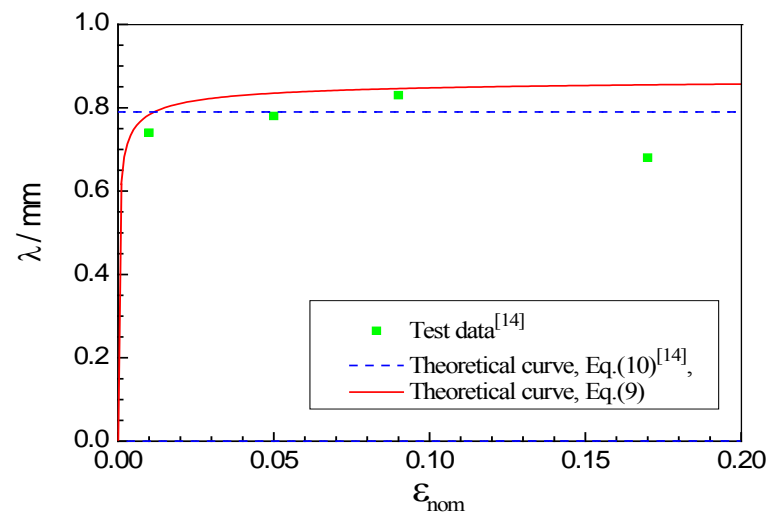

Figure 3. Half wavelength testing and calculated results. 


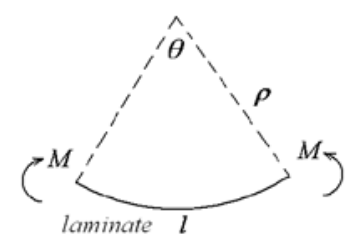

Figure 4. Relationship of packaged laminate.

The solid line and dotted line in Fig.3 are respectively given by Equations (9) and (10). It can be seen from Fig. 3 that the testing data are distributed at the neighborhood of the two theoretical curves. The half wavelength always exists and its value keeps constant through the whole bending process of EMC laminate for the dotted line. For the solid line the theoretical curve can divide two stages. when nominal strain is about less than 0.017 the half wavelength drastically increases with the increasing of bending level; when nominal strain is about greater than 0.017 the half wavelength fundamentally keeps constant. In fact, the fiber microbuckling in compressive zone still doesn't occur and bending behavior of EMC laminate still follow traditional linear elastic bending theory of unidirectional laminates at very little bending stage. In this study theoretical analysis is based on the condition that the fiber microbuckling has occurred, and so the partial theoretical solid line is not effective due to exceeding its serving range at very little bending stage. But the applicable and nonapplicable bending ranges of the theoretical model can approximately be recognized from the solid line and the critical strain is about 0.017 . The fact of gradually bending process of EMC laminate above $T_{\mathrm{g}}$ shows that Equation (9) is more reasonable than Equation (10).

\section{Nonlinear relation of bending moment and curvature}

The neutral axis location and half wavelength have already been solved in the above. Substituting the neutral axis location and half wavelength, Equations (1) and (9), into the total strain energy, Equation (7), according to the principal of virtual work the bending moment $M$ needed to bend the EMC laminate is

$$
\boldsymbol{M}=\frac{\partial U_{t}}{\partial \boldsymbol{\theta}}
$$

where $\theta$ is the central angle of the EMC laminate.

As schematically shown in Fig.4, the geometrical relationship of bending curvature $k$ and central angle $\theta$ of the packaged EMC laminate can be determined as

$$
\kappa=1 / \rho=\theta / l
$$

where $\rho$ is the curvature radius of packaged EMC laminate.

Combining Equation (11) with Equation (12), a new equation will be obtained as

$$
M=\frac{\partial U_{t}}{\partial \kappa} \frac{\partial \kappa}{\partial \theta}=\frac{1}{l} \frac{\partial U_{t}}{\partial \kappa}
$$

The following work will first be done before Equation (13) is solved. The neutral axis location and half wavelength, Equations (1) and (9), are substituted into each of strain energy expressions, Equations (3), (4), (5), (6) and (7) and the related values in Table 1 are also substituted into them. Fig. 5 that reveals the relationships of each of strain energies and bending curvature of EMC laminate can be plotted as the following. 


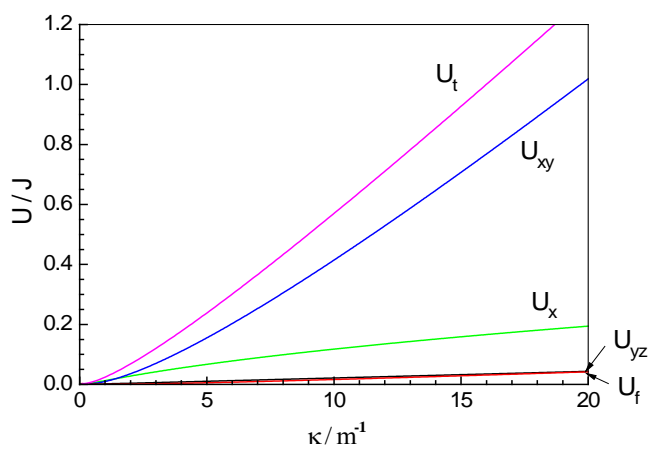

Figure 5. Relationships of strain energies and curvature.

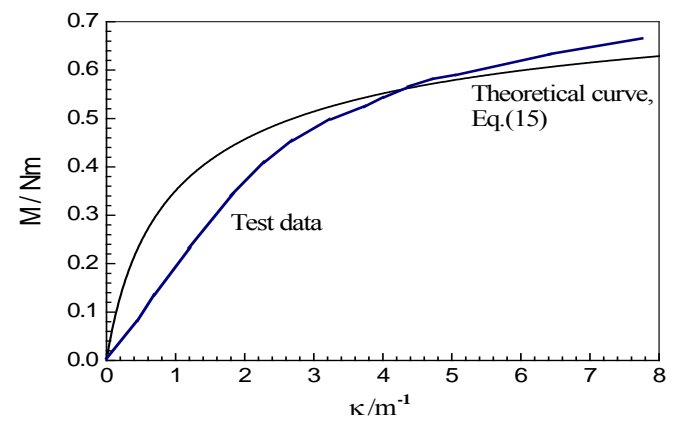

Figure 6. Curves of the moment versus curvature.

It is seen from Fig.5 that the total strain energy, $U_{t}$, is mainly composed of $U_{x x}$ and $U_{x y}$ and the other two strain energies, $\mathrm{U}_{\mathrm{yz}}$ and $\mathrm{U}_{\mathrm{f}}$, are very small and negligible. It is possible that that the fibers are very slender and their microbuckling bending level is also very small makes $U_{\mathrm{f}}$ very small. The amplitudes of fiber microbuckling are much less than half wavelengths and the micro varieties of amplitudes along the thickness of EMC laminate may lead to a very little energy $\mathrm{U}_{\mathrm{yz}}$. Hence, Equation (13) can be simplified as the following.

$$
M \approx \frac{1}{l}\left(\frac{\partial U_{x y}}{\partial \kappa}+\frac{\partial U_{x x}}{\partial \kappa}\right)
$$

The detailed derivation process of Equation (14) is treated as an appendix located at the back of the paper and the derived result of bending moment-curvature relationship is

$$
M=E_{x} w\left(\frac{G_{x y}}{E_{x}}\right)\left[\frac{t^{2}}{2}-\frac{\left(\frac{G_{x y}}{E_{x}}\right)^{2}}{3 \kappa^{2}}+\frac{\left(\frac{G_{x y}}{E_{x}}\right)^{3}+t \kappa\left(\frac{G_{x y}}{E_{x}}\right)^{2}-2 t^{2} \kappa^{2}\left(\frac{G_{x y}}{E_{x}}\right)}{3 \kappa^{2} \sqrt{\left(\frac{G_{x y}}{E_{x}}\right)^{2}+2 t \kappa\left(\frac{G_{x y}}{E_{x}}\right)}}\right]
$$

There are two curves in Fig.6. One is given via Equation (15) and the other is experimental curve. Of course, the related values in Table 1 should be substituted into Equation (15). The testing curve results from 4-point pure bending testing of EMC laminates. The specimens that are composed of shape memory epoxy and continuous IM7 carbon fibers are placed in an incubator that steadily keeps about $115^{\circ} \mathrm{C}$ during testing.

It can be seen from Fig. 2 that nominal bending strain $\varepsilon_{\text {nom }}$ is about 0.0018 when neutral axis starts to shift toward the tensile zone of EMC laminate. When the nominal bending strain $\varepsilon_{n o m}$ is less than 
0.0018 , microbuckling of the fibers in the compressive zone still doesn't occur and the neutral axis still locates the geometric midplane of EMC laminate. The traditional linear elastic bending theory of unidirectional laminates is applicable in such small bending stage. The theoretical investigation is not effective until nominal bending strain $\varepsilon_{n o m}$ is greater than 0.0018 in this paper. According to the formula, $\varepsilon_{\text {nom }}=t \kappa / 2$, the curvature $k$ corresponding to $\varepsilon_{\text {nom }}$ is $3.6 \mathrm{~m}^{-1}$. As schematically shown in Fig.6, when the curvature $k$ is less than $3.6 \mathrm{~m}^{-1}$ there are obviously discrepancies in the theoretical and testing curves. This can well be understood because the theoretical curve has already exceeded its applicable range and is regarded as invalidation. However, when curvature $k$ is greater than ${ }_{3.6} \mathrm{~m}^{-1}$ the theoretical curve is basically agreeable with the testing result. From this it can be seen that the theoretical analysis fundamentally meets the actual microbuckling bending process of an unidirectional EMC laminate above $T_{\mathrm{g}}$.

\section{Conclusions}

The neutral axis location and fiber microbuckling half wavelength are introduced, and at the same time the shortages are perfected when an EMC laminate is bent above the glass transition temperature of its resin $\left(T_{\mathrm{g}}\right)$. The results show that the applicable and nonapplicable curvature ranges of the theoretical model can approximately be recognized from the theoretical curve of half wavelength. Then, the known neutral axis location and half wavelength expressions are substituted into the total strain energy expression of packaged EMC laminate. According to the principal of virtual work, the total strain energy is deduced to yield the bending moment-curvature nonlinear relationship. The theoretical bending moment-curvature curve is basically agreeable with the 4-point pure bending testing result of EMC laminate during fiber microbuckling.

\section{Appendix: derivation of relationship between moment and curvature}

For the convenience, $a$ and $\Delta$ respectively denote as the following.

$$
a=\frac{G_{x y}}{E_{x}}, \Delta=\left(\frac{G_{x y}}{E_{x}}\right)^{2}+2 t \kappa\left(\frac{G_{x y}}{E_{x}}\right)
$$

Then, substituting Equation (A.1) into Equation (1) and the result is

$$
z_{n a}=t / 2+a / k--\sqrt{\Delta} / k
$$

A) Substituting Equation (A.2) into Equation (3), the result is determined as

$$
U_{x x}=\frac{1}{6} E_{x x} l w \frac{(\sqrt{\Delta}-a)^{3}}{\kappa}
$$

The following is derivation process of the partial derivative of Equation (A.3) with respect to curvature, $\kappa$.

$$
\begin{aligned}
& \frac{\partial \sqrt{\Delta}}{\partial \kappa}=\frac{\partial \sqrt{a^{2}+2 a t \kappa}}{\partial \kappa}=\frac{a t}{\sqrt{\Delta}} \\
& \frac{\partial}{\partial \kappa}(\sqrt{\Delta}-a)^{3}=\frac{3 a t(\sqrt{\Delta}-a)^{2}}{\sqrt{\Delta}}
\end{aligned}
$$




$$
\begin{array}{r}
\frac{\partial}{\partial \kappa}\left[\frac{(\sqrt{\Delta}-a)^{3}}{\kappa}\right]=\frac{1}{\kappa^{2}}\left[\frac{3 a t \kappa(\sqrt{\Delta}-a)^{2}}{\sqrt{\Delta}}-(\sqrt{\Delta}-a)^{3}\right] \\
\frac{1}{l} \frac{\partial U_{x x}}{\partial \kappa}=\frac{1}{6} E_{x x} w \cdot \frac{1}{\kappa^{2}} \cdot(\sqrt{\Delta}-a)^{2} \cdot\left(\frac{3 a t \kappa}{\sqrt{\Delta}}-\sqrt{\Delta}+a\right) \\
\frac{1}{l} \frac{\partial U_{x x}}{\partial \kappa}=w G_{x y} \cdot \frac{1}{6 a} \frac{1}{\kappa^{2}} \cdot(\sqrt{\Delta}-a)^{2} \cdot\left(\frac{3 a t \kappa}{\sqrt{\Delta}}-\sqrt{\Delta}+a\right) \\
\left(\frac{3 a t \kappa}{\sqrt{\Delta}}-\sqrt{\Delta}+a\right)=\frac{a t \kappa-a^{2}+a \sqrt{\Delta}}{\sqrt{\Delta}} \\
\frac{1}{l} \frac{\partial U_{x x}}{\partial \kappa}=w G_{x y} \cdot \frac{\left(a^{2}+a \kappa-t a \sqrt{\Delta}\right)(t \kappa-a+\sqrt{\Delta})}{3 \kappa^{2} \sqrt{\Delta}} \\
\frac{1}{l} \frac{\partial U_{x x}}{\partial \kappa}=w G_{x y} \cdot \frac{2 a^{2} \sqrt{\Delta}-2 a^{3}-2 a^{2} t \kappa+a t^{2} \kappa^{2}}{3 \kappa^{2} \sqrt{\Delta}}
\end{array}
$$

B) Substituting Equation (A.2) into Equation (4), the result is determined as

$$
U_{x y}=\frac{1}{2} G_{x y} l w \kappa\left(t+\frac{a}{\kappa}-\frac{\sqrt{\Delta}}{\kappa}\right)^{2}
$$

Similarly, the following is derivation process of the partial derivative of Equation (A.12) with respect to curvature, $k$.

$$
\begin{aligned}
& \frac{\partial}{\partial \kappa}\left[\kappa\left(t+\frac{a}{\kappa}-\frac{\sqrt{\Delta}}{\kappa}\right)^{2}\right]=\left(t+\frac{a}{\kappa}-\frac{\sqrt{\Delta}}{\kappa}\right)^{2}+\kappa \cdot 2\left(t+\frac{a}{\kappa}-\frac{\sqrt{\Delta}}{\kappa}\right) \cdot\left[\frac{-a^{2}}{\kappa^{2}}-\left(\frac{-a^{2}-a t \kappa}{\kappa^{2} \sqrt{\Delta}}\right)\right] \\
& \frac{\partial}{\partial \kappa}\left[\kappa\left(t+\frac{a}{\kappa}-\frac{\sqrt{\Delta}}{\kappa}\right)^{2}\right]=\left(t+\frac{a}{\kappa}-\frac{\sqrt{\Delta}}{\kappa}\right) \cdot\left(t+\frac{a}{\kappa}-\frac{\sqrt{\Delta}}{\kappa}+2 \kappa \cdot \frac{-a \sqrt{\Delta}+a^{2}+a t \kappa}{\kappa^{2} \sqrt{\Delta}}\right) \\
& t+\frac{a}{\kappa}-\frac{\sqrt{\Delta}}{\kappa}+2 \kappa \cdot \frac{-a \sqrt{\Delta}+a^{2}+a t \kappa}{\kappa^{2} \sqrt{\Delta}}=t+\frac{-a \kappa \sqrt{\Delta}+a^{2} \kappa}{\kappa^{2} \sqrt{\Delta}} \\
& \frac{1}{l} \frac{\partial U_{x y}}{\partial \kappa}=w G_{x y}\left[\frac{1}{2}\left(t+\frac{a}{\kappa}-\frac{\sqrt{\Delta}}{\kappa}\right)\left(t+\frac{-a \kappa \sqrt{\Delta}+a^{2} \kappa}{\kappa^{2} \sqrt{\Delta}}\right)\right] \\
& \frac{1}{l} \frac{\partial U_{x y}}{\partial \kappa}=w G_{x y} \frac{(t \kappa+a-\sqrt{\Delta})\left(t \kappa \sqrt{\Delta}+a^{2}-a \sqrt{\Delta}\right)}{2 \kappa^{2} \sqrt{\Delta}} \\
& \frac{1}{l} \frac{\partial U_{x y}}{\partial \kappa}=w G_{x y} \frac{t^{2} \kappa^{2} \sqrt{\Delta}-2 a^{2} \sqrt{\Delta}+2 a^{3}-2 a t^{2} \kappa^{2}+2 a^{2} t \kappa}{2 \kappa^{2} \sqrt{\Delta}}
\end{aligned}
$$

C) Substituting Equations (A.11) and (A.18) into Equation (14), the result is

$$
M=\frac{t^{2}}{2}-\frac{a^{2}}{3 \kappa^{2}}+\frac{a^{3}+a^{2} t \kappa-2 a t^{2} \kappa^{2}}{3 \kappa^{2} \sqrt{\Delta}}
$$

Then, substituting Equation (A.1) into (A.19), the result is Equation (15). 


\section{References}

1. Z D Wang, Z F Li, Z Y Xiong, R N Chang. Modeling Thermomechanical Behaviors of Shape Memory Polymer[J]. Applied Polymer Science, 2009, 113(1): 651 - 656

2. Y Liu. Thermomechanics of Shape Memory Polymers: Uniaxial Experiments and Constitutive Modeling[J]. Int. J. of Plasticity, 2004

3. Z Y Xiong, Z D Wang, Z F Li, R N Chang. Micromechanism of deformation in EMC laminates[J]. Materials Science and Engineering A, 2008, 496(4): 323-328

4. D Campbell, M S Lake, K Mallick.. A study of the compression mechanics of soft-resin composites[C]. 45th AIAA/ASME/ASCE/AHS/ASC Structures, Structural Dynamics and Materials Conference, Palm Springs, California, 2004

5. D Campbell, A K Maji. Deployment precision and mechanics of elastic memory composites [J]. AIAA Journal, AIAA 2003-1495

6. Z D Wang, Z F Li, Y S Wang. Micro-buckling solution of elastic memory laminates under bending [J]. JIMSS, 2009, 20:1565-1572

7. W H Francis, M S Lake, J S Hinkle. A review of classical fiber microbuckling analytical solutions for use with elastic memory composites [J]. AIAA Journal, AIAA 2006-1764

8. T W Murphey, T Meink, M M Mikulas. Some micromechanics considerations of the folding of rigidizable composite materials [J]. AIAA Journal, AIAA 2001-1418

9. K Call. Micro-mechanisms of Deformation in Fiber Reinforced Polymer Matrix Elastic Memory Composites [J]. AIAA Journal, AIAA 2001-1419

10. W H Francis, M S Lake, K Mallick, et al. Development and testing of a hinge/actuator incorporating elastic memory composites [J]. AIAA Journal, AIAA 2003-1496.

11. R Barret, D Campbell, M S Lake, et al. Development of a passively deployed rollout solar array [J]. AIAA Journal, AIAA 2006-4011

12. M R Schultz, M J Hulse, P N Keller. Neutrally stable composite tape springs [J]. AIAA Journal, AIAA 2006-1810

13. X Lan, Y J Liu, H B Lv, X H Wang, J S Leng and S Y Du. Fiber reinforced shape-memory polymer composite and its application in a deployable hinge [J]. Smart Material and Structures, 2009, 18 (2009) 024002 (6pp)

14. Zhao Wei, Liu LI Wu, Lan Xin. Study on application of shape-memory polymer composite in space deployable structures [J]. Manned Spaceflight. 2016, 22(5):594-601.

15. W H Francis, D Campbell, $M$ Lake, M Dunn, and H J Qi. Elastic Memory Composite Microbuckling Mechanics: Closed-Form Model with Empirical Correlation [J]. AIAA Journal, AIAA 2007-2164 